\title{
Robust Suboptimal Guaranteed Cost Control for 2-D Discrete Systems Described by Fornasini-Marchesini First Model
}

\author{
Manish Tiwari, Amit Dhawan \\ Electronics and Communication Engineering Department, Motilal Nehru National Institute of Technology, Allahabad, India. \\ Email: manishtiwari_me@yahoo.com,amit_dhawan2@rediffmail.com
}

Received December $20^{\text {th }}, 2011$; revised January $24^{\text {th }}, 2012$; accepted February $26^{\text {th }}, 2012$

\begin{abstract}
This paper considers the guaranteed cost control problem for a class of two-dimensional (2-D) uncertain discrete systems described by the Fornasini-Marchesini (FM) first model with norm-bounded uncertainties. New linear matrix inequality (LMI) based characterizations are presented for the existence of static-state feedback guaranteed cost controller which guarantees not only the asymptotic stability of closed loop systems, but also an adequate performance bound over all the admissible parameter uncertainties. Moreover, a convex optimization problem is formulated to select the suboptimal guaranteed cost controller which minimizes the upper bound of the closed-loop cost function.
\end{abstract}

Keywords: Guaranteed Cost Control; Linear Matrix Inequality; Lyapunov Methods; Robust Stability; 2-D Discrete Systems; Uncertain Systems

\section{Introduction}

In recent years, due to theoretical as well as application importance in the fields such as digital filtering, image processing, seismographic data processing, thermal processes, gas absorption, water stream heating etc. [1-8], the two-dimensional (2-D) systems have received considerable attention. The stability analysis of 2-D discrete systems described the Fornasini-Marchesini (FM) first model [9] have been investigated extensively [10-20]. In $[10-12,20]$, the method of nonnegative matrix theory has been proposed for the investigation of stability of 2-D systems described by the FM first model. Sufficient conditions for the asymptotic stability of 2-D systems described by the FM first model have been presented in [11-13]. In [12], it has been shown with the help of a counterexample that condition for the asymptotic stability given in [11] is incorrect and its corrected version is proposed. An improved Lyapunov based sufficient condition for the stability of 2-D linear systems described by the FM first model has been proposed in [14], and it is shown that the criterion given in [14] is less restrictive than those reported in $[11,12]$. A computationally attractive necessary and sufficient condition for the asymptotic stability of the FM first model has been derived in [15]. On the basis of the results given in [15], a connection has been established among structured singular value of a constant matrix and the stability, as well as the stability margin of the FM first model. Based on this connection, a novel sufficient condition for the asymptotic stability of the FM first model is obtained and it is shown by numerical simulations that the condition given in [15] is usually less conservative than that of [14]. In [16], the stability of 2-D periodically shift variant system represented by the FM first model has been studied. In [19], based on the sum-of-squares polynomials with matrix coefficients, an LMI based sufficient condition for asymptotic stability of 2-D systems described by the FM first model has been derived. Furthermore, it has been shown that the criterion given in [19] is more relaxed than those presented in $[15,18]$. In [20], necessary and sufficient conditions for the asymptotic stability of the FM first model using non-negative matrix theory have been proposed, and it has been shown that the conditions are sharper than those reported in $[10,11,17]$. Further, a survey of the existing literature on the stability of the 2-D discrete systems described by FM first model has been presented in [21].

The guaranteed cost control of uncertain 2-D discrete systems aims to design a robust controller to stabilize the closed loop system and mean while guarantee a specified level of performance index for all the admissible uncertainties. This issue has been discussed for uncertain 2-D discrete systems described by the FM second model [22-26] and Roesser model [27]. The guaranteed cost con- 
trol problem for uncertain 2-D discrete systems described by the FM first model is an important and challenging problem. However to the best of authors' knowledge, the guaranteed cost control problem for 2-D discrete uncertain systems described by the FM first model which is structurally distinct from FM second model and Roesser model has not been reported so far in the literature.

This paper, therefore, deals with the guaranteed cost control problem for uncertain 2-D discrete systems described by the FM first model with norm bounded uncertainties. The paper is organized as follows. The problem of robust guaranteed cost control for 2-D discrete uncertain systems described by the FM first model is formulated in Section 2. Some useful related results are also recalled in this section. In Section 3, we relate the notion of cost matrix to the quadratic stability and an upper bound on the closed-loop cost function. Sufficient conditions for the existence of static-state feedback guaranteed cost controllers are derived based on LMI approach. The static-state feedback guaranteed cost controllers are characterized by the feasible solution to a certain LMI. Further, a convex optimization problem is introduced to select the suboptimal guaranteed cost controller which minimizes the upper bound of the closed-loop cost function. An illustrative example showing the potential of the proposed technique is given in Section 4.

\section{Problem Formulation and Preliminaries}

Throughout the paper the following notations are used:

$\boldsymbol{R}^{n}$ real vector space of dimension $n$;

$\boldsymbol{R}^{n \times m}$ set of $n \times m$ real matrices;

$\mathbf{0}$ null matrix or null vector of appropriate dimension;

$\boldsymbol{I}$ identity matrix of appropriate dimension;

$\boldsymbol{G}^{T}$ transpose of matrix $\boldsymbol{G}$;

$\boldsymbol{G}>\mathbf{0}$ matrix $\boldsymbol{G}$ is positive definite symmetric;

$\boldsymbol{G}<\mathbf{0}$ matrix $\boldsymbol{G}$ is positive definite symmetric;

$\lambda_{\max }(\boldsymbol{G})$ maximum eigenvalue of matrix $\boldsymbol{G}$;

$\operatorname{diag}\{\cdots\}$ block diagonal matrix.

This paper deals with the problem of guaranteed cost control for a class of 2-D uncertain discrete systems described by the FM first model [9]. Specifically, the system under consideration is given by

$$
\begin{aligned}
\boldsymbol{x}(i+1, j+1) & =\left(\boldsymbol{A}_{1}+\Delta \boldsymbol{A}_{1}\right) \boldsymbol{x}(i, j+1) \\
& +\left(\boldsymbol{A}_{2}+\Delta \boldsymbol{A}_{2}\right) \boldsymbol{x}(i+1, j) \\
& +\left(\boldsymbol{A}_{3}+\Delta \boldsymbol{A}_{3}\right) \boldsymbol{x}(i, j) \\
& +(\boldsymbol{B}+\Delta \boldsymbol{B}) \boldsymbol{u}(i, j) \\
\boldsymbol{A} & =\left[\begin{array}{lll}
\boldsymbol{A}_{1} & \boldsymbol{A}_{2} & \boldsymbol{A}_{3}
\end{array}\right],
\end{aligned}
$$

where $\boldsymbol{x}(i, j)$ is an $n \times 1$ state vector, $A_{1} \in R^{n \times n}$, $\boldsymbol{A}_{2} \in R^{n \times n}, \quad \boldsymbol{A}_{3} \in R^{n \times n}, \boldsymbol{u}(i, j)$ is $m \times 1$ input vector, $\boldsymbol{B} \in R^{n \times m}$. The matrices $\Delta \boldsymbol{A}_{k}(k=1,2,3)$ and $\Delta \boldsymbol{B}$ represents parameter uncertainties which are assumed to be of the form

$$
\left[\begin{array}{cc}
\Delta \boldsymbol{A} & \Delta \boldsymbol{B}
\end{array}\right]=\boldsymbol{L} \boldsymbol{F}(i, j)\left[\begin{array}{ll}
\boldsymbol{E}_{1} & \boldsymbol{E}_{2}
\end{array}\right]
$$

where

$$
\begin{aligned}
& \Delta \boldsymbol{A}=\left[\begin{array}{lll}
\Delta \boldsymbol{A}_{1} & \Delta \boldsymbol{A}_{2} & \Delta \boldsymbol{A}_{3}
\end{array}\right], \\
& \boldsymbol{E}_{1}=\left[\begin{array}{lll}
\boldsymbol{E}_{11} & \boldsymbol{E}_{12} & \boldsymbol{E}_{13}
\end{array}\right] .
\end{aligned}
$$

In the above, $\boldsymbol{L} \in R^{n \times p}, \quad \boldsymbol{E}_{11} \in R^{q \times n}, \quad \boldsymbol{E}_{12} \in R^{q \times n}$, $\boldsymbol{E}_{13} \in R^{q \times n}$ and $\boldsymbol{E}_{2} \in R^{q \times m}$ can be regarded as known structural matrices of uncertainty and $\boldsymbol{F}(i, j) \in R^{p \times q}$ is an unknown matrix representing parameter uncertainty which satisfies

$$
\begin{aligned}
& \boldsymbol{F}^{T}(i, j) \boldsymbol{F}(i, j) \leq \boldsymbol{I} \\
& \text { (or equivalently, }\|\boldsymbol{F}(i, j)\| \leq 1) .
\end{aligned}
$$

Note that the uncertainty of (1c) satisfying (1f) has been widely adopted in robust control and filtering for 2-D uncertain systems [22-27].

It is assumed that the system (1a) has a finite set of initial conditions [24,25] i.e., there exist two positive integers $r_{1}$ and $r_{2}$ such that

$$
\boldsymbol{x}(i, 0)=0, i \geq r_{1} ; \boldsymbol{x}(0, j)=0, j \geq r_{2}
$$

and the initial conditions are arbitrary, but belong to the set [22-26]

$$
\begin{aligned}
S=\{ & \boldsymbol{x}(i, 0), \boldsymbol{x}(0, j) \in R^{n}: \boldsymbol{x}(i, 0)=\boldsymbol{M} \boldsymbol{N}_{1}, \quad 0 \leq i<r_{1} \\
& \left.\boldsymbol{x}(0, j)=\boldsymbol{M} \boldsymbol{N}_{2}, \quad 0 \leq j<r_{2}, \boldsymbol{N}_{k}^{T} \boldsymbol{N}_{k}<1 \quad(k=1,2)\right\}
\end{aligned}
$$

where $\boldsymbol{M}$ is a given matrix. Note that the vector $\boldsymbol{N}_{k}(k=1,2)$ can always be restricted as $\boldsymbol{N}_{k}^{T} \boldsymbol{N}_{k}<1$ $(k=1,2)$ by appropriately choosing $\boldsymbol{M}$. In other words, there is no loss of generality by choosing initial conditions as in (1h).

Associated with the uncertain system (1a) is the cost function:

$$
J=\sum_{i=0}^{\infty} \sum_{j=0}^{\infty}\left[\boldsymbol{u}^{T}(i, j) \boldsymbol{R} \boldsymbol{u}(i, j)+\boldsymbol{\xi}_{i j}^{T} \boldsymbol{W}_{1} \boldsymbol{\xi}_{i j}\right],
$$

where

$$
\begin{aligned}
& \boldsymbol{\xi}_{i j}=\left[\begin{array}{lll}
x^{T}(i, j+1) & \boldsymbol{x}^{T}(i+1, j) & \boldsymbol{x}^{T}(i, j)
\end{array}\right]^{T}, \\
& \mathbf{0}<\boldsymbol{R}=\boldsymbol{R}^{T} \in R^{m \times m}, \\
& \boldsymbol{W}_{1}=\operatorname{diag}\left\{\boldsymbol{Q}_{1}, \boldsymbol{Q}_{2}, \boldsymbol{Q}_{3}\right\}, \\
& \mathbf{0}<\boldsymbol{Q}_{k}=\boldsymbol{Q}_{k}{ }^{T} \in R^{n \times n} \quad(k=1,2,3) .
\end{aligned}
$$

We are interested in designing a static-state feedback control law 


$$
\boldsymbol{u}(i, j)=\boldsymbol{K}_{1} \boldsymbol{x}(i, j+1)+\boldsymbol{K}_{2} \boldsymbol{x}(i+1, j)+\boldsymbol{K}_{3} \boldsymbol{x}(i, j),(3)
$$

for the system (1) and the cost function (2), such that for all $\boldsymbol{F}(i, j)$ satisfying (1f), the resulting closed-loop system

$$
\begin{aligned}
\boldsymbol{x}(i+1, j+1)= & {\left[\left(\boldsymbol{A}_{1}+\Delta \boldsymbol{A}_{1}\right)+(\boldsymbol{B}+\Delta \boldsymbol{B}) \boldsymbol{K}_{1}\right] \boldsymbol{x}(i, j+1) } \\
& +\left[\left(\boldsymbol{A}_{2}+\Delta \boldsymbol{A}_{2}\right)+(\boldsymbol{B}+\Delta \boldsymbol{B}) \boldsymbol{K}_{2}\right] \boldsymbol{x}(i+1, j)(4) \\
& +\left[\left(\boldsymbol{A}_{3}+\Delta \boldsymbol{A}_{3}\right)+(\boldsymbol{B}+\Delta \boldsymbol{B}) \boldsymbol{K}_{3}\right] \boldsymbol{x}(i, j),
\end{aligned}
$$

is asymptotically stable and the closed loop value of the cost function

$$
J=\sum_{i=0}^{\infty} \sum_{j=0}^{\infty} \xi_{i j}^{T} W_{2} \xi_{i j}
$$

where

$$
\boldsymbol{W}_{2}=\boldsymbol{W}_{1}+\boldsymbol{K}^{T} \boldsymbol{R} \boldsymbol{K},
$$

and

$$
\boldsymbol{K}=\left[\begin{array}{lll}
\boldsymbol{K}_{1} & \boldsymbol{K}_{2} & \boldsymbol{K}_{3}
\end{array}\right],
$$

satisfies $J \leq J^{*}$, where $J^{*}$ is some specified constant.

Definition 2.1. Consider the system (1) and cost function (2), if there exist a control law $\boldsymbol{u}^{*}(i, j)$ and a positive scalar $J^{*}$ such that for all admissible uncertainties, the closed-loop system (4) is asymptotically stable and the closed-loop value of the cost function (5) satisfies $J \leq J^{*}$, then $J^{*}$ is said to be a guaranteed cost and $\boldsymbol{u}^{*}(i, j)$ is said to be a guaranteed cost control law for the uncertain system (1).

Now, we recall the following results.

Lemma 2.1. $[4,9,14]$ The 2-D discrete uncertain system (1) is globally asymptotically stable if and only if

$$
\begin{gathered}
\operatorname{det}\left\{\boldsymbol{I}-z_{1}\left(\boldsymbol{A}_{1}+\boldsymbol{L} \boldsymbol{F}(i, j) \boldsymbol{E}_{11}\right)-z_{2}\left(\boldsymbol{A}_{2}+\boldsymbol{L} \boldsymbol{F}(i, j) \boldsymbol{E}_{12}\right)\right. \\
\left.-z_{1} z_{2}\left(\boldsymbol{A}_{3}+\boldsymbol{L} \boldsymbol{F}(i, j) \boldsymbol{E}_{13}\right)\right\} \neq 0
\end{gathered}
$$

for $\left(z_{1}, z_{2}, \boldsymbol{F}(i, j)\right) \in \overline{U^{2}}$,

where

$$
\overline{U^{2}}=\left\{\left(z_{1}, z_{2}, \boldsymbol{F}(i, j)\right):\left|z_{1}\right| \leq 1,\left|z_{2}\right| \leq 1,\|\boldsymbol{F}(i, j)\| \leq 1\right\} .
$$

Remark 2.1. With $\Delta \boldsymbol{A}=\boldsymbol{L} \boldsymbol{F}(i, j)\left[\begin{array}{lll}\boldsymbol{E}_{11} & \boldsymbol{E}_{12} & \boldsymbol{E}_{13}\end{array}\right]=\mathbf{0}$, (6) is identified as global asymptotic stability condition ([14], Equation (3)) of the nominal system described by FM first model.

Now, as an extension of the result for the global asymptotic stability condition of the 2-D discrete FM first model given in [14], one can easily arrive at the following lemma.

Lemma 2.2. [14]. The uncertain system (4) is globally asymptotically stable, provided there exist $n \times n$ positive definite symmetric matrices $\boldsymbol{P}, \boldsymbol{P}_{1}$ and $\boldsymbol{P}_{2}$ such that

$$
\begin{aligned}
& \boldsymbol{\Gamma}=\left[\begin{array}{lll}
\boldsymbol{A}_{\Delta 1}+\boldsymbol{B}_{\Delta} \boldsymbol{K}_{1} & \boldsymbol{A}_{\Delta 2}+\boldsymbol{B}_{\Delta} \boldsymbol{K}_{2} & \boldsymbol{A}_{\Delta 3}+\boldsymbol{B}_{\Delta} \boldsymbol{K}_{3}
\end{array}\right]^{T} \\
& \times \boldsymbol{P}\left[\begin{array}{lll}
A_{\Delta 1}+B_{\Delta} K_{1} & A_{\Delta 2}+B_{\Delta} K_{2} & A_{\Delta 3}+B_{\Delta} K_{3}
\end{array}\right] \\
& -\left[\begin{array}{ccc}
\boldsymbol{P}_{1} & \mathbf{0} & \mathbf{0} \\
\mathbf{0} & \boldsymbol{P}_{2} & \mathbf{0} \\
\mathbf{0} & \mathbf{0} & \boldsymbol{P}-\boldsymbol{P}_{1}-\boldsymbol{P}_{2}
\end{array}\right]<\mathbf{0}
\end{aligned}
$$

for all $\|\boldsymbol{F}(i, j)\| \leq 1$,

where $\boldsymbol{A}_{\Delta 1}=\boldsymbol{A}_{1}+\Delta \boldsymbol{A}_{1}, \quad \boldsymbol{A}_{\Delta 2}=\boldsymbol{A}_{2}+\Delta \boldsymbol{A}_{2}, \boldsymbol{A}_{\Delta 3}=\boldsymbol{A}_{3}+\Delta \boldsymbol{A}_{3}$, and $\boldsymbol{B}_{\Delta}=\boldsymbol{B}+\Delta \boldsymbol{B}$.

On the basis of the above lemma, we have the following definition.

Definition 2.2. A state feedback controller. $\boldsymbol{u}(i, j)=\boldsymbol{K}_{1} \boldsymbol{x}(i, j+1)+\boldsymbol{K}_{2} \boldsymbol{x}(i+1, j)+\boldsymbol{K}_{3} \boldsymbol{x}(i, j)$ is said to define a quadratic guaranteed cost control associated with cost matrix $\mathbf{0}<\boldsymbol{P}=\boldsymbol{P}^{T} \in R^{n \times n}$ for the system (4) and cost function (5) if there exist a $3 n \times 3 n$ positive definite symmetric matrix $\boldsymbol{W}_{2}$ given by (5b) and $n \times n$ positive definite symmetric matrices $\boldsymbol{P}_{1}$ and $\boldsymbol{P}_{2}$ such that

$$
\boldsymbol{\Gamma}+\boldsymbol{W}_{2}<\mathbf{0} \text { for all }\|\boldsymbol{F}(i, j)\| \leq 1 .
$$

The following well-known lemma is needed in the proof of our main results.

Lemma 2.3. [22,25,27] Let $\boldsymbol{A} \in R^{n \times n}, \quad \boldsymbol{H} \in R^{n \times k}$, $\boldsymbol{E} \in R^{l \times n}$ and $\boldsymbol{Q}=\boldsymbol{Q}^{T} \in R^{n \times n}$ be given matrices. Then there exists a positive definite matrix $\boldsymbol{P}$ such that

$$
[\boldsymbol{A}+\boldsymbol{H} \boldsymbol{F} \boldsymbol{E}]^{T} \boldsymbol{P}[\boldsymbol{A}+\boldsymbol{H} \boldsymbol{F} \boldsymbol{E}]-\boldsymbol{Q}<\mathbf{0}
$$

for all $\boldsymbol{F}$ satisfying $\boldsymbol{F}^{T} \boldsymbol{F} \leq \boldsymbol{I}$, if and only if there exists a scalar $\varepsilon>0$ such that

$$
\left[\begin{array}{cc}
-\boldsymbol{P}^{-1}+\varepsilon \boldsymbol{H} \boldsymbol{H}^{T} & \boldsymbol{A} \\
\boldsymbol{A}^{T} & \varepsilon^{-1} \boldsymbol{E}^{T} \boldsymbol{E}-\boldsymbol{Q}
\end{array}\right]<\mathbf{0} .
$$

\section{Main Results}

In the following, we aim to relate the notion of cost matrix to the quadratic stability and an upper bound on the closed-loop cost function.

Lemma 3.1. Suppose there exists a cost matrix. $\mathbf{0}<\boldsymbol{P}=\boldsymbol{P}^{T} \in R^{n \times n}$ for the system (4) with initial conditions (1g), (1h) and cost function (5) such that (8) holds. Then, (i) system (4) is quadratically stable and (ii) the cost function satisfies the bound

$$
J \leq J^{*}=\left(r_{1}+r_{2}-1\right) \lambda_{\max }\left(\boldsymbol{M}^{T} \boldsymbol{P} \boldsymbol{M}\right)
$$

for all admissible parameter uncertainties.

Proof. Proof of (i) directly follows from Lemma 2.2 and Definition 2.2.

To prove (ii), consider a quadratic 2-D Lyapunov function:

$$
v(\boldsymbol{x}(i, j))=\boldsymbol{x}^{T}(i, j) \boldsymbol{P} \boldsymbol{x}(i, j)
$$

Let $\Delta v(\boldsymbol{x}(i, j))$ is defined as 


$$
\begin{aligned}
\Delta v(x(i, j))= & \boldsymbol{x}^{T}(i+1, j+1) \boldsymbol{P} \boldsymbol{x}(i+1, j+1)-\boldsymbol{x}^{T}(i, j+1) \boldsymbol{P}_{1} \boldsymbol{x}(i, j+1) \\
& -\boldsymbol{x}^{T}(i+1, j) \boldsymbol{P}_{2} \boldsymbol{x}(i+1, j)-\boldsymbol{x}^{T}(i, j)\left(\boldsymbol{P}-\boldsymbol{P}_{1}-\boldsymbol{P}_{2}\right) \boldsymbol{x}(i, j) .
\end{aligned}
$$

Along the trajectory of the closed-loop system (4), we obtain

$$
\Delta v(\boldsymbol{x}(i, j))=\boldsymbol{\xi}_{i j}^{T} \boldsymbol{\Gamma} \boldsymbol{\xi}_{i j},
$$

where $\boldsymbol{\xi}_{i j}^{T}$ and $\boldsymbol{\Gamma}$ are defined in (2b) and (7), respectively. Since $\boldsymbol{P}$ is a cost matrix, it follows from Definition 2.2 that

$$
\begin{aligned}
& J<-\sum_{i=0}^{\infty} \sum_{j=0}^{\infty} \Delta v(\boldsymbol{x}(i, j)) \\
& =-\sum_{i=0}^{\infty} \sum_{j=0}^{\infty}\left[\boldsymbol{x}^{T}(i+1, j+1) \boldsymbol{P} \boldsymbol{x}(i+1, j+1)-\boldsymbol{x}^{T}(i, j+1) \boldsymbol{P}_{1} \boldsymbol{x}(i, j+1)-\boldsymbol{x}^{T}(i+1, j) \boldsymbol{P}_{2} \boldsymbol{x}(i+1, j)-\boldsymbol{x}^{T}(i, j)\left(\boldsymbol{P}-\boldsymbol{P}_{1}-\boldsymbol{P}_{2}\right) \boldsymbol{x}(i, j)\right] \\
& +\sum_{i=0}^{\infty} \sum_{j=0}^{\infty}\left[\boldsymbol{x}^{T}(i, j) \boldsymbol{P} \boldsymbol{x}(i, j)-\boldsymbol{x}^{T}(i+1, j+1) \boldsymbol{P} \boldsymbol{x}(i+1, j+1)\right] \\
& =\sum_{i=1}^{r_{1}-1} \boldsymbol{x}^{T}(i, 0)\left(\boldsymbol{P}-\boldsymbol{P}_{1}\right) \boldsymbol{x}(i, 0)+\sum_{j=1}^{r_{2}-1} \boldsymbol{x}^{T}(0, j)\left(\boldsymbol{P}-\boldsymbol{P}_{2}\right) \boldsymbol{x}(0, j)+\boldsymbol{x}^{T}(0,0)\left(\boldsymbol{P}-\boldsymbol{P}_{1}-\boldsymbol{P}_{2}\right) \boldsymbol{x}(0,0) \\
& \boldsymbol{P}>\boldsymbol{P}_{1} \Rightarrow \boldsymbol{M}^{T} \boldsymbol{P} \boldsymbol{M} \geq \boldsymbol{M}^{T} \boldsymbol{P}_{1} \boldsymbol{M}, \\
& \boldsymbol{P}>\boldsymbol{P}_{2} \Rightarrow \boldsymbol{M}^{T} \boldsymbol{P} \boldsymbol{M} \geq \boldsymbol{M}^{T} \boldsymbol{P}_{2} \boldsymbol{M},
\end{aligned}
$$$$
=\sum_{i=0}^{\infty} \sum_{j=0}^{\infty}\left[\boldsymbol{x}^{T}(i, j+1) \boldsymbol{P}_{1} \boldsymbol{x}(i, j+1)-\boldsymbol{x}^{T}(i, j) \boldsymbol{P}_{1} \boldsymbol{x}(i, j)\right]+\sum_{i=0}^{\infty} \sum_{j=0}^{\infty}\left[\boldsymbol{x}^{T}(i+1, j) \boldsymbol{P}_{2} \boldsymbol{x}(i+1, j)-\boldsymbol{x}^{T}(i, j) \boldsymbol{P}_{2} \boldsymbol{x}(i, j)\right]
$$$$
=\sum_{i=1}^{\infty} \boldsymbol{x}^{T}(i, 0)\left(\boldsymbol{P}-\boldsymbol{P}_{1}\right) \boldsymbol{x}(i, 0)+\sum_{j=1}^{\infty} \boldsymbol{x}^{T}(0, j)\left(\boldsymbol{P}-\boldsymbol{P}_{2}\right) \boldsymbol{x}(0, j)+\boldsymbol{x}^{T}(0,0)\left(\boldsymbol{P}-\boldsymbol{P}_{1}-\boldsymbol{P}_{2}\right) \boldsymbol{x}(0,0)
$$$$
\leq\left[\left(r_{1}-1\right) \lambda_{\max }\left\{\boldsymbol{M}^{T}\left(\boldsymbol{P}-\boldsymbol{P}_{1}\right) \boldsymbol{M}\right\}+\left(r_{2}-1\right) \lambda_{\max }\left\{\boldsymbol{M}^{T}\left(\boldsymbol{P}-\boldsymbol{P}_{2}\right) \boldsymbol{M}\right\}+\lambda_{\max }\left\{\boldsymbol{M}^{T}\left(\boldsymbol{P}-\boldsymbol{P}_{1}-\boldsymbol{P}_{2}\right) \boldsymbol{M}\right\}\right],
$$

where use has been made of (5), (1g) and (1h) and the relation $\lim _{i+j \rightarrow \infty} \boldsymbol{x}(i, j)=\mathbf{0}$. Note that if (8) holds, we have

and

$$
\boldsymbol{P}>\left(\boldsymbol{P}_{1}+\boldsymbol{P}_{2}\right) \Rightarrow \boldsymbol{M}^{T} \boldsymbol{P} \boldsymbol{M} \geq \boldsymbol{M}^{T}\left(\boldsymbol{P}_{1}+\boldsymbol{P}_{2}\right) \boldsymbol{M} .
$$

Therefore, the upper bound in (11) can be obtained by applying (18) in (17). This completes the proof of the Lemma 3.1.
The following theorem establishes that the problem of determining guaranteed cost control for system (4) and the cost function (5) can be recast to an LMI feasibility problem.

Theorem 3.1. Consider system (4) with initial conditions (1g), (1h) and cost function (5), then there exists a static-state feedback controller $\boldsymbol{u}(i, j)=\boldsymbol{K}_{1} \boldsymbol{x}(i, j+1)+\boldsymbol{K}_{2} \boldsymbol{x}(i+1, j)+\boldsymbol{K}_{3} \boldsymbol{x}(i, j)$ that solves the addressed robust guaranteed cost control problem if there exist a positive scalar $\mathcal{E}, m \times n$ matrices $\boldsymbol{U}_{1}, \boldsymbol{U}_{2}, \boldsymbol{U}_{3}$ and $n \times n$ positive definite symmetric matrices $\boldsymbol{V}, \boldsymbol{R}_{1}, \boldsymbol{R}_{2}$ such that the following LMI is feasible:

$$
\left[\begin{array}{ccccccccc}
-\boldsymbol{V}+\boldsymbol{L} \boldsymbol{L}^{T} & \boldsymbol{A}_{1} \boldsymbol{V}+\boldsymbol{B} \boldsymbol{U}_{1} & \boldsymbol{A}_{2} \boldsymbol{V}+\boldsymbol{B} \boldsymbol{U}_{2} & \boldsymbol{A}_{3} \boldsymbol{V}+\boldsymbol{B} \boldsymbol{U}_{3} & \mathbf{0} & \mathbf{0} & \mathbf{0} & \mathbf{0} & \mathbf{0} \\
\left(\boldsymbol{A}_{1} \boldsymbol{V}+\boldsymbol{B} \boldsymbol{U}_{1}\right)^{T} & -\boldsymbol{R}_{1} & \mathbf{0} & \mathbf{0} & \boldsymbol{V} \boldsymbol{Q}_{1}^{1 / 2} & \boldsymbol{U}_{1}^{T} \boldsymbol{R}^{1 / 2} & \mathbf{0} & \mathbf{0} & \overline{\boldsymbol{E}}_{11}^{T} \\
\left(\boldsymbol{A}_{2} \boldsymbol{V}+\boldsymbol{B} \boldsymbol{U}_{2}\right)^{T} & \mathbf{0} & -R_{2} & \mathbf{0} & \mathbf{0} & \boldsymbol{U}_{2}^{T} \boldsymbol{R}^{1 / 2} & \boldsymbol{V} \boldsymbol{Q}_{2}^{1 / 2} & \mathbf{0} & \overline{\boldsymbol{E}}_{12}^{T} \\
\left(\boldsymbol{A}_{3} \boldsymbol{V}+\boldsymbol{B} \boldsymbol{U}_{3}\right)^{T} & \mathbf{0} & \mathbf{0} & -\boldsymbol{V}+\boldsymbol{R}_{1}+\boldsymbol{R}_{2} & \mathbf{0} & \boldsymbol{U}_{3}^{T} \boldsymbol{R}^{1 / 2} & \mathbf{0} & \boldsymbol{V} \boldsymbol{Q}_{3}^{1 / 2} & \overline{\boldsymbol{E}}_{13}^{T} \\
\mathbf{0} & \boldsymbol{Q}_{1}^{T / 2} \boldsymbol{V} & \mathbf{0} & \mathbf{0} & -\varepsilon \boldsymbol{I} & \mathbf{0} & \mathbf{0} & \mathbf{0} & \mathbf{0} \\
\mathbf{0} & \boldsymbol{R}^{T / 2} \boldsymbol{U}_{1} & \boldsymbol{R}^{T / 2} \boldsymbol{U}_{2} & \boldsymbol{R}^{T / 2} \boldsymbol{U}_{3} & \mathbf{0} & -\varepsilon \boldsymbol{I} & \mathbf{0} & \mathbf{0} & \mathbf{0} \\
\mathbf{0} & \mathbf{0} & \boldsymbol{Q}_{2}^{T / 2} \boldsymbol{V} & \mathbf{0} & \mathbf{0} & \mathbf{0} & -\varepsilon \boldsymbol{I} & \mathbf{0} & \mathbf{0} \\
\mathbf{0} & \mathbf{0} & \mathbf{0} & \boldsymbol{Q}_{3}^{T / 2} \boldsymbol{V} & \mathbf{0} & \mathbf{0} & \mathbf{0} & -\varepsilon \boldsymbol{I} & \mathbf{0} \\
\mathbf{0} & \overline{\boldsymbol{E}}_{11} & \overline{\boldsymbol{E}}_{12} & \overline{\boldsymbol{E}}_{13} & \mathbf{0} & \mathbf{0} & \mathbf{0} & \mathbf{0} & -\boldsymbol{I}
\end{array}\right]
$$


where

$$
\overline{\boldsymbol{E}}_{11}=\left(\boldsymbol{E}_{11} \boldsymbol{V}+\boldsymbol{E}_{2} \boldsymbol{U}_{1}\right), \overline{\boldsymbol{E}}_{12}=\left(\boldsymbol{E}_{12} \boldsymbol{V}+\boldsymbol{E}_{2} \boldsymbol{U}_{2}\right), \overline{\boldsymbol{E}}_{13}=\left(\boldsymbol{E}_{13} \boldsymbol{V}+\boldsymbol{E}_{2} \boldsymbol{U}_{3}\right)
$$

In this situation, the suitable control laws are given by

$$
\begin{gathered}
\boldsymbol{K}_{1}=\boldsymbol{U}_{1} \boldsymbol{V}^{-1}, \\
\boldsymbol{K}_{2}=\boldsymbol{U}_{2} \boldsymbol{V}^{-1},
\end{gathered}
$$

and

$$
\boldsymbol{K}_{3}=\boldsymbol{U}_{3} \boldsymbol{V}^{-1}
$$

Moreover, the closed-loop cost function satisfies the following bound

$$
J^{*}=\left(r_{1}+r_{2}-1\right) \varepsilon \lambda_{\max }\left(\boldsymbol{M}^{T} \boldsymbol{V}^{-1} \boldsymbol{M}\right) .
$$

Proof. Using 1(c)-1(f), 5(b) and Lemma 2.3, (8) can be rearranged as

$$
\begin{gathered}
\left.\begin{array}{cc}
-\boldsymbol{P}^{-1}+\varepsilon^{-1} \boldsymbol{L} \boldsymbol{L}^{T} & \left(\boldsymbol{A}_{1}+\boldsymbol{B} \boldsymbol{K}_{1}\right) \\
\left(\boldsymbol{A}_{1}+\boldsymbol{B} \boldsymbol{K}_{1}\right)^{T} & -\boldsymbol{P}_{1}+\boldsymbol{Q}_{1}+\boldsymbol{K}_{1}^{T} \boldsymbol{R} \boldsymbol{K}_{1}+\varepsilon\left(\boldsymbol{E}_{11}+\boldsymbol{E}_{2} \boldsymbol{K}_{1}\right)^{T}\left(\boldsymbol{E}_{11}+\boldsymbol{E}_{2} \boldsymbol{K}_{1}\right) \\
\left(\boldsymbol{A}_{2}+\boldsymbol{B} \boldsymbol{K}_{2}\right)^{T} & \boldsymbol{K}_{2}^{T} \boldsymbol{R} \boldsymbol{K}_{1}+\varepsilon\left(\boldsymbol{E}_{12}+\boldsymbol{E}_{2} \boldsymbol{K}_{2}\right)^{T}\left(\boldsymbol{E}_{11}+\boldsymbol{E}_{2} \boldsymbol{K}_{1}\right) \\
\left(\boldsymbol{A}_{3}+\boldsymbol{B} \boldsymbol{K}_{3}\right)^{T} & \boldsymbol{K}_{3}^{T} \boldsymbol{R} \boldsymbol{K}_{1}+\varepsilon\left(\boldsymbol{E}_{13}+\boldsymbol{E}_{2} \boldsymbol{K}_{3}\right)^{T}\left(\boldsymbol{E}_{11}+\boldsymbol{E}_{2} \boldsymbol{K}_{1}\right) \\
\left(\boldsymbol{A}_{2}+\boldsymbol{B} \boldsymbol{K}_{2}\right) & \left(\boldsymbol{A}_{3}+\boldsymbol{B} \boldsymbol{K}_{3}\right) \\
\boldsymbol{K}_{1}^{T} \boldsymbol{R} \boldsymbol{K}_{2}+\varepsilon\left(\boldsymbol{E}_{11}+\boldsymbol{E}_{2} \boldsymbol{K}_{1}\right)^{T}\left(\boldsymbol{E}_{12}+\boldsymbol{E}_{2} \boldsymbol{K}_{2}\right) & \boldsymbol{K}_{1}^{T} \boldsymbol{R} \boldsymbol{K}_{3}+\varepsilon\left(\boldsymbol{E}_{11}+\boldsymbol{E}_{2} \boldsymbol{K}_{1}\right)^{T}\left(\boldsymbol{E}_{13}+\boldsymbol{E}_{2} \boldsymbol{K}_{3}\right) \\
-\boldsymbol{P}_{2}+\boldsymbol{Q}_{2}+\boldsymbol{K}_{2}^{T} \boldsymbol{R} \boldsymbol{K}_{2}+\varepsilon\left(\boldsymbol{E}_{12}+\boldsymbol{E}_{2} \boldsymbol{K}_{2}\right)^{T}\left(\boldsymbol{E}_{12}+\boldsymbol{E}_{2} \boldsymbol{K}_{2}\right) & \boldsymbol{K}_{2}^{T} \boldsymbol{R} \boldsymbol{K}_{3}+\varepsilon\left(\boldsymbol{E}_{12}+\boldsymbol{E}_{2} \boldsymbol{K}_{2}\right)^{T}\left(\boldsymbol{E}_{13}+\boldsymbol{E}_{2} \boldsymbol{K}_{3}\right) \\
\boldsymbol{K}_{3}^{T} \boldsymbol{R} \boldsymbol{K}_{2}+\varepsilon\left(\boldsymbol{E}_{13}+\boldsymbol{E}_{2} \boldsymbol{K}_{3}\right)^{T}\left(\boldsymbol{E}_{12}+\boldsymbol{E}_{2} \boldsymbol{K}_{2}\right) & -\boldsymbol{P}+\boldsymbol{P}_{1}+\boldsymbol{P}_{2}+\boldsymbol{Q}_{3}+\boldsymbol{K}_{3}^{T} \boldsymbol{R} \boldsymbol{K}_{3}+\varepsilon\left(\boldsymbol{E}_{13}+\boldsymbol{E}_{2} \boldsymbol{K}_{3}\right)^{T}\left(\boldsymbol{E}_{13}+\boldsymbol{E}_{2} \boldsymbol{K}_{3}\right)
\end{array}\right]
\end{gathered}
$$

Pre- and post-multiplying (22) by the $\operatorname{diag}\left\{\varepsilon^{1 / 2} \boldsymbol{I}, \varepsilon^{1 / 2} \boldsymbol{P}^{-1}, \varepsilon^{1 / 2} \boldsymbol{P}^{-1}, \varepsilon^{1 / 2} \boldsymbol{P}^{-1}\right\}$ yields

$$
\left.\begin{array}{ccc}
-\boldsymbol{V}+\boldsymbol{L} \boldsymbol{L}^{T} & \left(\boldsymbol{A}_{1} V+\boldsymbol{B} U_{1}\right) & \left(\boldsymbol{A}_{2} V+\boldsymbol{B} \boldsymbol{U}_{2}\right) \\
\left(\boldsymbol{A}_{1} V+\boldsymbol{B} U_{1}\right)^{T} & -\boldsymbol{R}_{1}+\varepsilon^{-1} \boldsymbol{V} \boldsymbol{Q}_{1} \boldsymbol{V}+\varepsilon^{-1} \boldsymbol{U}_{1}^{T} \boldsymbol{R} \boldsymbol{U}_{1}+\overline{\boldsymbol{E}}_{11}^{T} \overline{\boldsymbol{E}}_{11} & \varepsilon^{-1} \boldsymbol{U}_{1}^{T} \boldsymbol{R} \boldsymbol{U}_{2}+\overline{\boldsymbol{E}}_{11}^{T} \overline{\boldsymbol{E}}_{12} \\
\left(\boldsymbol{A}_{2} V+\boldsymbol{B} U_{2}\right)^{T} & \varepsilon^{-1} \boldsymbol{U}_{2}^{T} \boldsymbol{R} \boldsymbol{U}_{1}+\overline{\boldsymbol{E}}_{12}^{T} \overline{\boldsymbol{E}}_{11} & -\boldsymbol{R}_{2}+\varepsilon^{-1} \boldsymbol{V} \boldsymbol{Q}_{2} \boldsymbol{V}+\varepsilon^{-1} \boldsymbol{U}_{2}^{T} \boldsymbol{R} \boldsymbol{U}_{2}+\overline{\boldsymbol{E}}_{12}^{T} \overline{\boldsymbol{E}}_{12} \\
\left(\boldsymbol{A}_{3} V+\boldsymbol{B} U_{3}\right)^{T} & \varepsilon^{-1} \boldsymbol{U}_{3}^{T} \boldsymbol{R} \boldsymbol{U}_{1}+\overline{\boldsymbol{E}}_{13}^{T} \overline{\boldsymbol{E}}_{11} & \varepsilon^{-1} \boldsymbol{U}_{3}^{T} \boldsymbol{R} \boldsymbol{U}_{2}+\overline{\boldsymbol{E}}_{13}^{T} \overline{\boldsymbol{E}}_{12} \\
& \left(\boldsymbol{A}_{3} \boldsymbol{V}+\boldsymbol{B} \boldsymbol{U}_{3}\right) \\
\varepsilon^{-1} \boldsymbol{U}_{1}^{T} \boldsymbol{R} \boldsymbol{U}_{3}+\overline{\boldsymbol{E}}_{11}^{T} \overline{\boldsymbol{E}}_{13} \\
\varepsilon^{-1} \boldsymbol{U}_{2}^{T} \boldsymbol{R} \boldsymbol{U}_{3}+\overline{\boldsymbol{E}}_{12}^{T} \overline{\boldsymbol{E}}_{13} \\
-\boldsymbol{V}+\boldsymbol{R}_{1}+\boldsymbol{R}_{2}+\varepsilon^{-1} \boldsymbol{V} \boldsymbol{Q}_{3} \boldsymbol{V}+\varepsilon^{-1} \boldsymbol{U}_{3}^{T} \boldsymbol{R} \boldsymbol{U}_{3}+\overline{\boldsymbol{E}}_{13}^{T} \overline{\boldsymbol{E}}_{13}
\end{array}\right]<\mathbf{0 ,},
$$

where

$$
\boldsymbol{V}=\varepsilon \boldsymbol{P}^{-1}
$$

and

$$
\varepsilon^{-1} \boldsymbol{V} \boldsymbol{P}_{1} \boldsymbol{V}=\boldsymbol{R}_{1}>\mathbf{0}, \varepsilon^{-1} \boldsymbol{V} \boldsymbol{P}_{2} \boldsymbol{V}=\boldsymbol{R}_{2}>\mathbf{0} .
$$

The equivalence of (23) and (19) follows trivially from the schur complements. Using (24), the bound of cost function can be easily obtained from (11). This completes the proof of the Theorem 3.1.

Remark 3.1. Note that the matrix inequality (19) is linear in variables $\varepsilon, \boldsymbol{U}_{1}, \boldsymbol{U}_{2}, \boldsymbol{U}_{3}, \boldsymbol{V}, \boldsymbol{R}_{1}$ and $\boldsymbol{R}_{2}$ which can be easily solved using Matlab LMI Toolbox $[28,29]$.

Remark 3.2. It is clear that the upper bound on the closed-loop cost function is dependent on the choice of the guaranteed cost controllers. In particular, the guaran- teed cost controller that renders the corresponding guaranteed cost upper bound as small as possible is more interesting; such a controller is said to be an optimal guaranteed cost controller. Apparently, the upper bound (21) is not a convex function in $\boldsymbol{V}$ and $\varepsilon$. Hence, finding the minimum of this upper bound cannot be considered as a convex optimization problem. Since $\varepsilon$ and $\lambda_{\max }\left(\boldsymbol{M}^{T} \boldsymbol{V}^{-1} \boldsymbol{M}\right)$ are positive, we may obtain a suboptimal guaranteed cost controller by minimizing $\varepsilon+\lambda_{\max }\left(\boldsymbol{M}^{T} \boldsymbol{V}^{-1} \boldsymbol{M}\right)$. Based on Theorem 3.1, the design problem of such a suboptimal guaranteed cost controller can be formulated as an optimization problem.

Theorem 3.2. Consider system (4) with initial conditions (1g), (1h) and cost function (5), then there exists a suboptimal static-state feedback controller 
$\boldsymbol{u}(i, j)=\boldsymbol{K}_{1} \boldsymbol{x}(i, j+1)+\boldsymbol{K}_{2} \boldsymbol{x}(i+1, j)+\boldsymbol{K}_{3} \boldsymbol{x}(i, j)$ that solves the addressed robust guaranteed cost control problem if the following optimization problem

$$
\begin{aligned}
& \text { minimize }(\varepsilon+\lambda) \\
& \text { s.t. }\left\{\begin{array}{l}
(\text { i). }(19), \\
\text { (ii). }\left[\begin{array}{cc}
-\lambda \boldsymbol{I} & \boldsymbol{M}^{T} \\
\boldsymbol{M} & -\boldsymbol{V}
\end{array}\right]<\mathbf{0},
\end{array}\right.
\end{aligned}
$$

has a feasible solution $\lambda>0, \varepsilon>0, m \times n$ matrices $\boldsymbol{U}_{1}, \boldsymbol{U}_{2}, \boldsymbol{U}_{3}$ and $n \times n$ positive definite symmetric matrices $\boldsymbol{V}, \boldsymbol{R}_{1}, \boldsymbol{R}_{2}$. In this situation, the suboptimal control laws are $\boldsymbol{K}_{1}=\boldsymbol{U}_{1} \boldsymbol{V}^{-1}, \boldsymbol{K}_{2}=\boldsymbol{U}_{2} \boldsymbol{V}^{-1}$ and $\boldsymbol{K}_{3}=\boldsymbol{U}_{3} \boldsymbol{V}^{-1}$ which ensures the minimization of the guaranteed cost in (21).

Proof. By Theorem 3.1, the control laws (20) constructed in terms of any feasible solution $\varepsilon, \boldsymbol{V}, \boldsymbol{R}_{1}$, $\boldsymbol{R}_{2}, \boldsymbol{U}_{1}, \boldsymbol{U}_{2}$ and $\boldsymbol{U}_{3}$ are the guaranteed cost controllers of system (4). To obtain the optimum value of the upper bound of guaranteed cost, the term $\lambda_{\text {max }}\left(\boldsymbol{M}^{T} \boldsymbol{V}^{-1} \boldsymbol{M}\right)$ in (21) is changed to $\lambda_{\text {max }}\left(\boldsymbol{M}^{T} \boldsymbol{V}^{-1} \boldsymbol{M}\right)<\lambda \Leftrightarrow \boldsymbol{M}^{T} \boldsymbol{V}^{-1} \boldsymbol{M}<\lambda \boldsymbol{I}$ which, in turn, implies the constraint (ii) in (26). Thus, the minimization of $(\varepsilon+\lambda)$ implies the minimization of the guaranteed cost in (21). This completes the proof of Theorem 3.2.

\section{Illustrative Example}

In this section, we will give a specific example to demonstrate the effectiveness of Theorem 3.2. Consider a 2-D discrete uncertain system given by (1) and (2) with

$$
\begin{aligned}
& \boldsymbol{A}_{1}=\left[\begin{array}{cc}
0 & -0.7500 \\
-1.0000 & 0
\end{array}\right], \boldsymbol{A}_{2}=\left[\begin{array}{cc}
0.2700 & 0 \\
0 & 0
\end{array}\right], \\
& \boldsymbol{A}_{3}=\left[\begin{array}{cc}
0 & -0.0010 \\
0 & 0
\end{array}\right], \boldsymbol{B}=\left[\begin{array}{l}
0.1000 \\
0.2000
\end{array}\right], \boldsymbol{L}=\left[\begin{array}{l}
0 \\
1
\end{array}\right], \\
& \boldsymbol{E}_{11}=\left[\begin{array}{ll}
-0.050 & -0.007
\end{array}\right], \boldsymbol{E}_{12}=\left[\begin{array}{ll}
-0.060 & -0.002
\end{array}\right], \\
& \boldsymbol{E}_{13}=\left[\begin{array}{ll}
0.1200 & 0.0010
\end{array}\right], \boldsymbol{E}_{2}=[0.0075], \\
& \boldsymbol{R}=\left[\begin{array}{ll}
0.0060
\end{array}\right], r_{1}=r_{2}=2, \boldsymbol{Q}_{1}=\operatorname{diag}\{6,6\}, \\
& \boldsymbol{Q}_{2}=\operatorname{diag}\{0.66,0.66\}, \boldsymbol{Q}_{3}=\operatorname{diag}\{0.044,0.044\}, \\
& \boldsymbol{M}=\left[\begin{array}{l}
0.0100 \\
0.0800
\end{array}\right] .
\end{aligned}
$$

Using Lemma 2.1, it is easy to verify that the above system is unstable. We wish to construct a suitable guaranteed cost controller for this system, such that the corresponding cost bound is minimized. To this end, we apply our proposed method (Theorem 3.2) to find the suboptimal guaranteed cost controller. It is found using the Matlab LMI toolbox [28,29] that the optimization problem (26) is feasible for the present example and the optimal solution is given by

$$
\begin{aligned}
& \boldsymbol{V}=\left[\begin{array}{ll}
2.0644 & 1.1173 \\
1.1173 & 1.6047
\end{array}\right], \boldsymbol{R}_{1}=\left[\begin{array}{ll}
1.0533 & 0.5442 \\
0.5442 & 1.2592
\end{array}\right] \\
& \boldsymbol{R}_{2}=\left[\begin{array}{ll}
0.9315 & 0.5168 \\
0.5168 & 0.3114
\end{array}\right], \boldsymbol{U}_{1}=\left[\begin{array}{ll}
11.0423 & 3.1939
\end{array}\right], \\
& \boldsymbol{U}_{2}=\left[\begin{array}{ll}
2.0681 & 1.1193
\end{array}\right], \boldsymbol{U}_{3}=\left[\begin{array}{ll}
-0.0041 & -0.0060
\end{array}\right] \\
& \varepsilon=34.1593, \lambda=0.0056 .
\end{aligned}
$$

By Theorem 3.2, the suboptimal guaranteed cost controllers to this system are

$$
\begin{aligned}
& \boldsymbol{K}_{1}=\left[\begin{array}{ll}
6.8548 & -2.7825
\end{array}\right], \quad \boldsymbol{K}_{2}=\left[\begin{array}{ll}
1.0018 & -0.0000
\end{array}\right], \\
& \boldsymbol{K}_{3}=\left[\begin{array}{ll}
0.0001 & -0.0038
\end{array}\right],
\end{aligned}
$$

and the least upper bound of the corresponding closed loop cost function is $J^{*}=0.5773$.

\section{Conclusion}

In this paper, we have presented a solution to the guaranteed cost control problem for a class of uncertain 2-D discrete systems described by the FM first model in a LMI framework. The existence condition for the staticstate feedback guaranteed cost controller has been derived in terms of a certain LMI. The parameterized representation of a set of guaranteed cost controllers (if they exist) has been presented in terms of the feasible solutions to the LMI. Finally, a convex optimization problem has been introduced to design the suboptimal guaranteed cost controller that minimizes the upper bound of the closed-loop cost function.

\section{REFERENCES}

[1] A. T. Kaczorek, "Two-Dimensional Linear Systems," Springer-Verlag, Berlin, 1985.

[2] R. N. Bracewell, "Two-Dimensional Imaging," PrenticeHall Signal Processing Series, Prentice-Hall, Englewood Cliffs, 1995.

[3] W.-S. Lu and A. Antoniou, "Two-Dimensional Digital Filters," Marcel Dekker, New York, 1992.

[4] N. K. Bose, “Applied Multidimensional System Theory," Van Nostrand Reinhold, New York, 1982.

[5] W. Marszalek, "Two-Dimensional State-Space Discrete Models for Hyperbolic Partial Differential Equations," Applied Mathematical Modelling, Vol. 8, No. 1, 1984, pp. 11-14. doi:10.1016/0307-904X(84)90170-7

[6] C. Du, L. Xie and C. Zhang, " $H_{\infty}$ Control and Robust Stabilization of Two-Dimensional Systems in Roesser Models," Automatica, Vol. 37, No. 2, 2001, pp. 205-211. doi:10.1016/S0005-1098(00)00155-2 
[7] J. S.-H. Tsai, J. S. Li and L.-S. Shieh, "Discretized Quadratic Optimal Control for Continuous-Time Two-Dimensional Systems," IEEE Transactions on Circuits and Systems I, Vol. 49, No. 1, 2002, pp. 116-125.

[8] R. Yang, L. Xie and C. Zhang, " $H_{2}$ and Mixed $H_{2} / H_{\infty}$ Control of Two-Dimensional Systems in Roesser Model," Automatica, Vol. 42, No. 9, 2006, pp. 1507-1514. doi:10.1016/j.automatica.2006.04.002

[9] E. Fornasini and G. Marchesini, "State-Space Realization Theory of Two Dimensional Filters," IEEE Transactions on Automatic Control, Vol. 21, No. 4, 1976, pp. 484-492. doi:10.1109/TAC.1976.1101305

[10] G.-D. Hu and M. Liu, "Simple Criteria for Stability of Two-Dimensional Linear Systems," IEEE Transactions on Signal Processing, Vol. 53, No. 12, 2005, pp. 4720-4723. doi:10.1109/TSP.2005.859265

[11] T. Bose and D. A. Trautman, "Two's Complement Quantization in Two-Dimensional State-Space Digital Filters," IEEE Transactions on Signal Processing, Vol. 40, No. 10, 1992, pp. 2589-2592. doi:10.1109/78.157299

[12] Y. Su and A. Bhaya, "On the Bose-Trautman Condition for Stability of Two-Dimensional Linear Systems," IEEE Transactions on Signal Processing, Vol. 46, No. 7, 1998, pp. 2069-2070. doi:10.1109/78.700987

[13] T. Bose, "Stability of 2-D State-Space System with Overflow and Quantization," IEEE Transactions on Circuits and Systems II, Vol. 42, No. 6, 1995, pp. 432-434. doi:10.1109/82.392319

[14] H. Kar and V. Singh, "Stability of 2-D Systems Described by Fornasini-Marchesini First Model," IEEE Transactions on Signal Processing, Vol. 51, No. 6, 2003, pp. 16751676. doi:10.1109/TSP.2003.811237

[15] T. Zhou, "Stability and Stability Margin for a Two-Dimensional System," IEEE Transactions on Signal Processing, Vol. 54, No. 9, 2006, pp. 3483-3488. doi:10.1109/TSP.2006.879300

[16] R. Thamvichai and T. Bose, "Stability of 2-D Periodically Shift Variant Filters," IEEE Transactions on Circuits and Systems II, Vol. 49, No. 1, 2002, pp. 61-64. doi:10.1109/82.996060

[17] A. Bhaya, E. Kaszkurewicz and Y. Su, "Stability of Asynchronous Two-Dimensional Fornasini-Marchesini Dynamical Systems," Linear Algebra and Its Application, Vol. 332, 2001, pp. 257-263. doi:10.1016/S0024-3795(00)00317-7

[18] D. Henrion, M. Sebek and O. Bachelier, "Rank-1 LMI Approach to Stability of 2-D Polynomial Matrices," Mul- tidimens Systems Signal Process, Vol. 12, No. 11, 2001, pp. 33-48. doi:10.1023/A:1008464726878

[19] B. Dumitrescu, "LMI Stability Tests for the FornasiniMarchesini Model," IEEE Transactions on Signal Processing, Vol. 56, No. 8, 2008, pp. 4091-4095.

[20] T. Liu, "Stability Analysis of Linear 2-D Systems," Signal Processing, Vol. 88, No. 8, 2008, pp. 2078-2084. doi:10.1016/j.sigpro.2008.02.007

[21] M. Tiwari and A. Dhawan, "A Survey on Stability of 2-D Discrete Systems Described by Fornasini-Marchesini First Model," Proceedings of the International Conference on Power Control and Embedded Systems, Allahabad, 2010, pp. 1-4. doi:10.1109/ICPCES.2010.5698674

[22] X. Guan, C. Long and G. Duan, "Robust Optimal Guaranteed Cost Control for 2D Discrete Systems," IET-Control Theory \& Applications, Vol. 148, No. 5, 2001, pp. 355-361.

[23] A. Dhawan and H. Kar, "Comment on Robust Optimal Guaranteed Cost Control for 2-D Discrete Systems," IETControl Theory \& Applications, Vol. 1, No. 4, 2007, pp. 1188-1190.

[24] A. Dhawan and H. Kar, "LMI-Based Criterion for the Robust Guaranteed Cost Control of 2-D Systems Described by the Fornasini-Marchesini Second Model," Signal Processing, Vol. 87, No. 3, 2007, pp. 479-488. doi:10.1016/j.sigpro.2006.06.002

[25] A. Dhawan and H. Kar, "Optimal Guaranteed Cost Control of 2-D Discrete Uncertain Systems: An LMI Approach," Signal Processing, Vol. 87, No. 12, 2007, pp. 3075-3085. doi:10.1016/j.sigpro.2007.06.001

[26] A. Dhawan and H. Kar, "LMI Approach to Suboptimal Guaranteed Cost Control for 2-D Discrete Uncertain Systems," Journal of Signal and Information Processing, Vol. 2, No. 4, 2011, pp. 292-300. doi:10.4236/jsip.2011.24042

[27] A. Dhawan and H. Kar, "An LMI Approach to Robust Optimal Guaranteed Cost Control of 2-D Discrete Systems Described by the Roesser Model," Signal Processing, Vol. 90, No. 9, 2010, pp. 2648-2654. doi:10.1016/j.sigpro.2010.03.008

[28] S. Boyd, L. El Ghaoui, E. Feron and V. Balakrishnan, "Linear Matrix Inequalities in System and Control Theory," SIAM, Philadelphia, 1994. doi:10.1137/1.9781611970777

[29] P. Gahinet, A. Nemirovski, A. J. Laub and M. Chilali, "LMI Control Toolbox-For Use with Matlab," The MATH Works Inc., Natick, 1995. 